\title{
Changes in lung volume and ventilation following transition from invasive to noninvasive respiratory support and prone positioning in preterm infants
}

\author{
Pauline S. van der Burg ${ }^{1}$, Martijn Miedema ${ }^{1}$, Frans H. de Jongh' ${ }^{1}$, Inez Frerichs ${ }^{2}$ and Anton H. van Kaam
}

\begin{abstract}
BACKGROUND: To minimize secondary lung injury, ventilated preterm infants are extubated as soon as possible. To maximize extubation success, they are often placed in prone position. The effect of extubation and subsequent prone positioning on lung volumes is currently unknown.
\end{abstract}

METHODS: Changes in end-expiratory lung volume ( $\triangle \mathrm{EELV}$ ), tidal volume $\left(V_{T}\right)$, and ventilation distribution were monitored during transition from endotracheal to nasal continuous positive airway pressure and following prone positioning using electrical impedance tomography. In addition, the continuous distending pressure (CDP) and oxygen need $\left(\mathrm{FiO}_{2}\right)$ were recorded.

RESULTS: Twenty preterm infants (GA 28.7 $\pm 1.7 \mathrm{wk}$ ) were included. Following extubation, the CDP decreased from $7.9 \pm 0.5$ to $6.0 \pm 0.2 \mathrm{cmH}_{2} \mathrm{O}$, while the $\mathrm{FiO}_{2}$ remained stable. Both $\triangle \mathrm{EELV}$ and $V_{\mathrm{T}}$ increased significantly $(P<0.05)$ after extubation, without changing ventilation distribution. Prone positioning resulted in a further increase in $\triangle \mathrm{EELV}(P<0.01)$ and a decrease in respiratory rate. $V_{T}$ remained stable but its distribution clearly shifted toward the ventral lung regions.

CONCLUSION: Infants who are transitioned from invasive to noninvasive respiratory support are able to maintain their EELV and increase their $V_{T}$. Prone positioning increases EELV and shifts tidal ventilation to the ventral lung regions. The latter suggests that infants should preferably be placed in prone position after extubation.

$\mathbf{P}$ reterm infants suffering from respiratory distress syndrome have a reduced end-expiratory lung volume (EELV) and lung compliance, leading to impaired ventilation and oxygenation (1). A substantial proportion of these preterm infants needs to be intubated and mechanically ventilated (2). Despite the beneficial effect on lung function and gas exchange, mechanical ventilation also has a downside as it can lead to secondary lung injury and subsequent bronchopulmonary dysplasia $(3,4)$. For this reason, preterm infants are extubated as soon as possible and transferred back to noninvasive respiratory support, predominantly nasal continuous positive airway pressure
(nCPAP). To make these transitions successful, infants should be able to maintain an optimal EELV and normal ventilation during nCPAP. Placing infants in prone position might facilitate this transition to nCPAP as this posture seems to increase EELV and improve respiratory mechanics (5-8).

Despite the fact that EELV might play an important role in successful transition from invasive to noninvasive respiratory support, the changes in lung volume following extubation have been poorly investigated. Only two studies have assessed changes in functional residual capacity before and after extubation, but these changes were not measured continuously and the infants were extubated to a head box and not nCPAP $(9,10)$. Moreover, the role of positioning was not addressed in these studies.

The fact that lung volume changes have been poorly studied in preterm infants is mainly caused by the lack of easily applicable, inexpensive, methods to measure lung volume continuously at bedside, which do not alter respiratory mechanics or increase dead space in this vulnerable population. Electrical impedance tomography (EIT) is a relatively new, noninvasive technique, which measures changes in lung electrical impedance in a crosssectional slice of the chest that correlate well with changes in aeration (11). Furthermore, EIT also provides information on regional changes in volume and ventilation (12-14). Recent studies have shown that EIT is feasible in the preterm population and that the lung volume changes measures in the examined slice are representative for the whole lung (15-17).

We have therefore conducted an observational study using EIT to gain more insight into the (regional) changes in EELV and ventilation following transition from invasive to noninvasive respiratory support in premature infants with respiratory distress syndrome. Furthermore, we have assessed the additional effect of prone position shortly after extubation on these parameters.

\section{RESULTS}

We included a convenience sample of 20 infants with a mean gestational age of $28.7 \pm 1.7 \mathrm{wk}$ and birth weight of $1,125 \pm 388 \mathrm{~g}$. Patient characteristics are shown in Table 1 . Two

'Department of Neonatology, Emma Children's Hospital, Academic Medical Center, Amsterdam, The Netherlands; ${ }^{2}$ Department of Anesthesiology and Intensive Care Medicine, University Medical Center Schleswig-Holstein, Campus Kiel, Germany. Correspondence: Pauline S. van der Burg (p.s.burgvander@amc.uva.nl) 
Table 1. Patient characteristics

\begin{tabular}{|c|c|c|c|c|}
\hline Subject number & $\begin{array}{c}\text { Gestational } \\
\text { age (wk) }\end{array}$ & Weight (g) & $\begin{array}{l}\text { 5'Apgar } \\
\text { score }\end{array}$ & $\begin{array}{l}\text { Age } \\
\text { (days) }\end{array}$ \\
\hline 1 & 29.0 & 970 & 10 & 1 \\
\hline 2 & 32.0 & 1,070 & 9 & 3 \\
\hline 3 & 28.0 & 720 & 8 & 1 \\
\hline 4 & 29.3 & 1,095 & 8 & 1 \\
\hline 5 & 30.9 & 1,310 & 8 & 2 \\
\hline 6 & 26.4 & 750 & 6 & 2 \\
\hline 7 & 25.0 & 750 & 7 & 1 \\
\hline 8 & 29.0 & 1,360 & 8 & 1 \\
\hline 9 & 29.6 & 1,060 & 10 & 2 \\
\hline 10 & 26.9 & 1,040 & 7 & 1 \\
\hline 11 & 28.7 & 1190 & 7 & 2 \\
\hline 12 & 28.3 & 1,040 & 8 & 1 \\
\hline 13 & 29.7 & 885 & 7 & 3 \\
\hline 14 & 29.9 & 1,375 & 9 & 1 \\
\hline 15 & 30.7 & 2,300 & 8 & 1 \\
\hline 16 & 28.1 & 1,010 & 9 & 1 \\
\hline 17 & 26.3 & 895 & 8 & 1 \\
\hline 18 & 27.9 & 565 & 8 & 1 \\
\hline 19 & 29.7 & 1,655 & 9 & 2 \\
\hline 20 & 28.9 & 1,455 & 8 & 1 \\
\hline Median & 28.9 & 1,050 & 8 & 1 \\
\hline
\end{tabular}

patients did not tolerate high-frequency oscillatory ventilation (HFOV) without oscillations and in two patients the electrodes detached when turning them from supine to prone positioning, leaving only 18 measurements at these time points. The continuous distending pressure (CDP) before extubation, after extubation, and after switching to prone position was, respectively, $7.9 \pm 0.5,6.0 \pm 0.2$, and $6.0 \pm 0.2 \mathrm{cmH}_{2} \mathrm{O}$. The $\mathrm{FiO}_{2}$ remained stable at these three time points (Table 2).

Compared to ET-CPAP, EIT showed a significant increase in EELV (median $3.8 \mathrm{AU} / \mathrm{kg}$, interquartile range: -0.8-27.4 $\mathrm{AU} / \mathrm{kg}, P<0.05)$ after extubation to nCPAP in supine position. The increase in EELV was located mostly in the dorsal lung regions (Figure 1). In all patients, the EELV stabilized within the 10-min measurement period. Both respiratory rate and $V_{\mathrm{T}}$ increased after extubation, but only the latter reached statistical significance. In response to these changes, $\mathrm{TcPCO}_{2}$ showed a modest, nonsignificant reduction and $\mathrm{SpO} 2$ a modest but significant increase (Table 2). Ventilation distribution did not change after extubation (Table 2; Figure 2).

After changing to prone position, EELV significantly increased compared to nCPAP in the supine position (median: 36.0 , interquartile range: $23.7-63.3 \mathrm{AU} / \mathrm{kg}, P<0.01$ ) (Figure 1). Again, the increase in EELV favored the dorsal lung regions, although there was also an increase observed in the ventral regions (Figure 1). The positional change resulted in a significant decrease in respiratory rate compared to supine position with no change in $V_{\mathrm{T}}$ and $\mathrm{TcPCO}_{2}$ (Table 2). $\mathrm{SpO}_{2}$ remained
Table 2. Ventilatory parameters

\begin{tabular}{lccc}
\hline & $\begin{array}{c}\text { ET-CPAP, supine } \\
\text { position }(n=18)\end{array}$ & $\begin{array}{c}\text { nCPAP, supine } \\
\text { position }(n=20)\end{array}$ & $\begin{array}{c}\text { nCPAP, prone } \\
\text { position }(n=18)\end{array}$ \\
\hline $\mathrm{RR}\left(\mathrm{min}^{-1}\right)$ & $60.3 \pm 18.2$ & $68.5 \pm 9.5$ & $62.8 \pm 14.1^{\dagger}$ \\
$\mathrm{TcPCO}_{2}(\mathrm{kPa})$ & $6.7 \pm 1.5$ & $6.4 \pm 2.1$ & $6.3 \pm 1.9$ \\
$V_{\mathrm{T}}(\mathrm{AU} / \mathrm{kg})^{\mathrm{a}}$ & $7.3(5.1-9.3)$ & $11.7(8.0-13.8)^{*}$ & $10.8(9.2-13.6)$ \\
$\mathrm{SpO}_{2}(\%)^{\mathrm{a}}$ & $92.0(80.0-98.0)$ & $96.0(94.0-99.3)^{* *}$ & $96.5(93.8-98.0)$ \\
$\mathrm{F}_{\mathrm{i}}(\%)^{\mathrm{a}}$ & $21.0(21.0-25.0)$ & $21.0(21.0-24.3)$ & $21.0(21.0-24.0)$ \\
$\mathrm{AUC}_{\text {ant }}(\%)$ & $47.9 \pm 8.0$ & $47.8 \pm 5.1$ & $54.1 \pm 4.8^{++}$ \\
$\mathrm{CoV}(\%)$ & $51.4 \pm 4.6$ & $51.1 \pm 3.0$ & $47.6 \pm 3.1^{++}$ \\
\hline
\end{tabular}

Data are presented as mean \pm SD or median (interquartile range: $25-75$ ) when stated differentlya. Comparative analyses between ET-CPAP and nCPAP in supine position, and between nCPAP supine and nCPAP in prone position were performed using student $t$-test or Wilcoxon rank sum test when appropriate.

AUC, area under the curve of the ventral lung region; $\mathrm{CoV}$, geometric center of ventilation; $\mathrm{nCPAP}$, nasal continuous positive airway pressure; RR, respiratory rate. *Indicates a significant change compared to ET-CPAP, $P<0.05$. ** Indicates a significant change compared to ET-CPAP, $P<0.01$. Indicates a significant change compared to nCPAP, supine position, $P<0.05 .{ }^{+}$Indicates a significant change compared to nCPAP, supine position, $P<0.001$

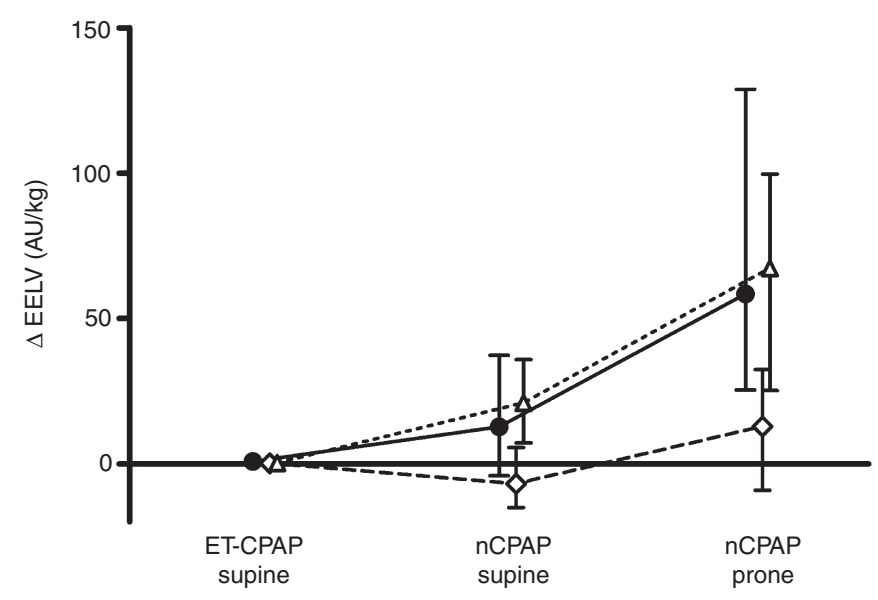

Figure 1. Changes in end-expiratory lung volume, global (solid line, closed circles), ventral (dashed line, open diamonds), and dorsal (dotted line, open triangles). Data are presented as median with interquartile range.

stable after switching to prone position. Ventilation significantly increased in the ventral regions as indicated by significant changes in area under the curve and geometrical center of ventilation (Table 2).

\section{DISCUSSION}

This study shows that preterm infants are able to maintain their EELV when transitioned from endotracheal to nasal CPAP. Placing these infants in prone position results in an increase in EELV and a ventral shift in tidal ventilation distribution.

To our knowledge, this is the first study that has continuously monitored changes in EELV and ventilation during transition from invasive to noninvasive respiratory support. To avoid any interference of mechanical ventilation with these parameters, we stopped the oscillations during HFOV, making extubation a true transition from endotracheal to nasal CPAP. According to our local protocol, infants were extubated to a nasal CDP 


\section{Articles | van der Burgetal.}

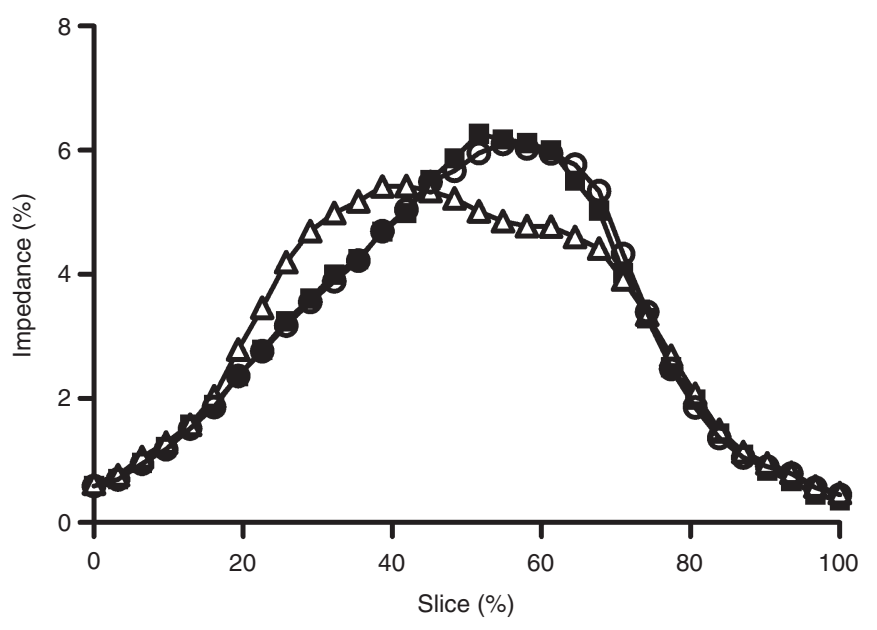

Figure 2. Percentage of tidal impedance change normalized to body weight in 32 anterior-to-posterior chest slices at different study phases; endotracheal continuous positive airway pressure (CPAP) (closed squares) in supine position, nasal CPAP in supine position (open circles) and nCPAP in prone position (open triangles). The slices were denoted by setting the most anterior part to $0 \%$ and the most posterior part to $100 \%$. Data are presented as mean values.

$2 \mathrm{cmH}_{2} \mathrm{O}$ lower than during HFOV, because it has been suggested that the alveolar CDP delivered by the SensorMedics 3100 ventilator is $1-2 \mathrm{cmH}_{2} \mathrm{O}$ lower than the pressure recorded at the hub of the endotracheal tube when delivered with an inspiratory time of 33\%, as is custom in our unit (18-20). Despite this lower CDP EELV remained stable after extubation. This finding is probably best explained by the fact that the infant regains control of the upper airways and laryngeal regulation after removal of the endotracheal tube. Previous studies have shown that the upper airways play an important role in maintaining functional residual capacity in infants $(21,22)$. The fact that tidal volumes also increased after extubation may also have contributed to the stability of the EELV.

EIT also allowed us to assess the regional changes in EELV following extubation. Although the changes in EELV were relatively small, it was interesting to observe that the dorsal (dependent) but not the ventral (nondependent) lung regions showed a modest increase in EELV. We can only speculate on possible explanations for this finding. First, spontaneous (negative pressure) breathing with control of the upper airways may have a different effect on EELV in the dependent lung regions compared to breathing through an endotracheal tube supported by positive airway pressures. Second, the absence of endotracheally delivered positive airway pressure following extubation may have increased venous return to the heart. This in turn may have limited an increase in lung aeration and thus lung impedance in the ventral part of the chest, as blood has a five times lower impedance than lung tissue at expiration (23).

Many infants on noninvasive support are placed in the prone position as previous studies have demonstrated that this reduces thoraco-abdominal asynchrony, improves oxygenation and increases functional residual capacity $(5,7,24)$. Our study also shows an increase in EELV when switching from supine to prone position but adds important information on its regional distributions. It shows that the increase in EELV is most prominent in the dorsal regions (25), which may imply a gravitational effect resulting in an increased aeration in these now nondependent regions. Although this cannot be substantiated by the present study, this effect on aeration in the dorsal regions may be accompanied by a reduction in blood and fluid content.

In addition to changes in EELV, we have also assessed the effect of extubation and body positioning on oxygenation and ventilation. The changes in EELV following extubation and prone positioning only had a small impact on oxygenation, suggesting that the changes in EELV were mainly caused by distension and not recruitment. The low $\mathrm{FiO}_{2}$ also seems to support this assumption. Removal of the endotracheal tube resulted in a significant increase in $V_{\mathrm{T}}$, which is probably best explained by the reduction in airway resistance $(26,27)$. This increase in $V_{\mathrm{T}}$ resulted in only a small, nonsignificant, reduction in $\mathrm{TcPCO}_{2}$. This indicates that airway dead space may have also increased after extubation due to the slight increase in EELV. Placing the infant in prone position did not change tidal volume but did result in a significant reduction in respiratory rate compared to supine position. As $\mathrm{Tc} \mathrm{PCO}_{2}$ remained stable this suggests more efficient breathing, probably caused by a reduction in thoraco-abdominal asynchrony (7).

In the present study, we have also determined the distribution of tidal ventilation. The paradigm that ventilation distribution in infants follows a reversed pattern compared to adults with a preferable distribution to the nondependent (uppermost) lung has recently been challenged (28-31). It has been suggested that this pattern only applies to sedated and mechanically ventilated infants. In spontaneously breathing infants, ventilation is distributed much more evenly throughout the lung $(28,29)$. Our study showed that application of CDP via the endotracheal or nasal route did not impact ventilation distribution in these spontaneously breathing preterm infants. Comparable to other studies $(28,30)$, ventilation slightly favored the dorsal lung regions. However, and in contrast to a previous study (30), placing the infants in prone position resulted in a clear shift in ventilation distribution to the ventral, dependent lung regions. It is unclear why our findings are different from these previous studies. It might be that the lung behaves different to positional change in the first hours after extubation to nCPAP. A previous report in a term born infant seems to support this hypothesis as ventilation distribution clearly shifted to the ventral region after placing this infant in prone position $1 \mathrm{~h}$ after extubation (15). Whether this shift in ventilation distribution to the ventral lung regions is consistent over time cannot be answered by this study.

Our study has several limitations that need to be addressed. First, the positioning of the infants during and after extubation was not randomized. Extubation in our unit is routinely performed in supine positioning as this provides the best access for airway management and placement of nCPAP. For this reason, the nursing staff was not comfortable extubating infants in prone position. In theory, the increase in EELV following prone positioning may have been an ongoing improvement 
after extubation, not related to the positional change. The fact that the EELV stabilized within the 10-min measurement period after extubation and the increase in EELV is consistent with previous studies makes this very unlikely. Second, this study only addressed the immediate changes in EELV and ventilation, using 10-min measurement periods. Whether these effects are maintained over a longer period of time needs further study. Third, this study only included patients with respiratory distress syndrome, extubated in the first $72 \mathrm{~h}$ after birth. The results might differ when dealing with other causes of respiratory failure or older infants. Finally, this study does not offer insight into absolute lung volume changes as it is not yet possible to calibrate the impedance changes during HFOV. However, this does not compromise the results of this study as we were interested in relative and not absolute changes in EELV and ventilation.

In conclusion, this study shows that preterm infants are able to maintain their EELV when transitioned from invasive to noninvasive respiratory support. Furthermore, it reveals that prone positioning increases EELV and provides more efficient ventilation, making this the preferred position following extubation in preterm infants.

\section{METHODS}

The study was performed in the neonatal intensive care unit of the Emma Children's Hospital, Academic Medical Center (Amsterdam, The Netherlands), where preterm infants $(<37 \mathrm{wk})$ with a suspected diagnosis of respiratory distress syndrome and failing nCPAP are treated with open lung HFOV. All patients are ventilated in the supine position and are not sedated or paralyzed. Infants were included in the study if they were eligible for extubation according to our local protocol, i.e., a CDP $\leq 8 \mathrm{cmH}_{2} \mathrm{O}$ and a fraction of inspired oxygen $\left(\mathrm{FiO}_{2}\right) \leq 0.30$. Exclusion criteria were congenital anomalies of the chest and/or abdomen and a fragile skin condition. Written informed consent was obtained from both parents and the study was approved by the Institutional Review Board of the Academic Medical Center, Amsterdam, The Netherlands.

\section{Patient Data Collection}

Patient characteristics including gestational age, birth weight, postnatal age, and Apgar scores were collected. Together with the EIT recordings, $\mathrm{FiO}_{2}$, heart rate, transcutaneous oxygen saturation $\left(\mathrm{SpO}_{2}\right)$, and transcutaneous partial pressure of carbon dioxide $\left(\mathrm{TcPCO}_{2}\right)$ were registered throughout the experiment.

\section{Study Protocol}

The study consisted of two phases during which impedance changes were continuously measured with EIT. During the first phase, within $10 \mathrm{~min}$ before extubation, patients were switched from HFOV (SensorMedics 3100, CareFusion, Yorba Linda, CA) to endotracheal CPAP (ET-CPAP) for $2 \mathrm{~min}$ prior to extubation by stopping the oscillations and allowing for spontaneous breathing. Next, the patients were extubated to nCPAP, delivered by the Infant Flow SiPAP system (CareFusion) with a CDP of $6 \mathrm{cmH}_{2} \mathrm{O}$. Following extubation, the impedance changes were registered for $10 \mathrm{~min}$ in supine position.

During the second phase, initiated $10 \mathrm{~min}$ after extubation, the infants were transferred from supine to prone position. Lung volume and ventilation changes were again measured for 10 min using EIT.

\section{EIT Data Collection}

Before extubation, 16 hand-trimmed electrocardiography electrodes (Blue Sensor, BRS-50-K, Ambu, Linthicum Heights, MD) were placed equidistantly on the thorax circumference, just above the nipple line and connected to the Goettingen Goe-MF II EIT system (CareFusion). Repetitive electrical currents $(5 \mathrm{~mA}, 100 \mathrm{kHz})$ were injected cyclically (scan rate: $44 \mathrm{~Hz}$ ) through adjacent electrode pairs. Voltage changes were registered between all other passive adjacent electrode pairs. A back-projection algorithm generated a series of $32 \times 32$ pixel matrices of relative impedance changes $(\Delta \mathrm{Z})$ by comparing these voltage changes to a reference period. Impedance changes were registered using the Veit software (CareFusion). Data analysis was performed off-line with AUSPEX version 1.6 (VUmc, Amsterdam, The Netherlands) after all data collection was completed.

\section{EIT Data Analysis}

As EIT cannot measure absolute changes in lung volumes, the change in EELV $(\triangle \mathrm{EELV})$ was calculated by selecting a stable 30 -s period at the start of the study protocol, which was used as reference for the subsequent recordings. Next, the relative changes in EELV were calculated for each step, by averaging the relative $\Delta \mathrm{Z}$ at the troughs of the spontaneous breaths in a stable 30 -s recording period at the end of each step to allow for lung volume to reach equilibrium. $\triangle E E L V$ was then normalized for body weight.

EIT-derived tidal volume $\left(V_{\mathrm{T}}\right)$ changes were calculated for each examination phase by referencing the selected 30 -s period to the average $\Delta \mathrm{Z}$ in that period. The EIT waveforms of relative $\Delta \mathrm{Z}$ values were then band-pass filtered in the band of spontaneous breathing rate $(10 / \mathrm{min}$ below the actual breathing frequency and $10 / \mathrm{min}$ above its second harmonic). The peaks and troughs of the signal were identified and averaged over the selected period. The average amplitude was normalized for body weight.

Regional ventilation distribution of the spontaneous breaths was assessed by means of functional EIT (fEIT) images. fEIT images were generated by calculating the SD of the impedance waveforms in each individual pixel within the $32 \times 32$ pixel matrix. A ventilation profile was derived from each fEIT image showing the distribution of ventilation in 32 anteroposterior slices, as previously described (32). Subsequently, the areas under the curve (AUC) for the ventral or anterior (slice 1-16, $\mathrm{AUC}_{\mathrm{ant}}$ ) and dorsal or posterior (slice 17-32) regions were calculated. The AUC ant describes the proportion of the total tidal impedance changes in the anterior lung regions. $\mathrm{An} \mathrm{AUC}_{\text {ant }}$ less than $50 \%$ indicates a posterior oriented and more than $50 \%$ an anterior oriented distribution of tidal ventilation. Finally, the geometrical center of ventilation was determined as an additional measure describing the ventilation distribution $(32,33)$. The geometrical center of ventilation represents the level in the anterior-posterior axis (0-100\%) where the AUC of tidal impedance changes is identical in the lung regions above and below this level. A geometrical center of ventilation more than $50 \%$ indicates a posterior oriented and less than $50 \%$ an anterior oriented distribution of tidal ventilation.

\section{Statistical Analysis}

Statistical analysis was performed using GraphPad Prism 5.0 (GraphPad Software, San Diego, CA) and SPSS version 20.0 (SPSS, Chicago, IL). Data were expressed as mean with SD or median with interquartile range, as appropriate. Comparative analysis was performed using the Wilcoxon signed rank test for skewed data and the paired student $t$-test for normally distributed data.

\section{ACKNOWLEDGMENT}

The work was performed at the Department of Neonatology, Emma Children's Hospital, Academic Medical Center, Amsterdam, The Netherlands.

Disclosures: This work was financially supported by Chiesi Pharmaceutical, Parma, Italy. The EIT device was kindly provided by CareFusion, Yorba Linda, CA.

\section{REFERENCES}

1. Jobe AH, Ikegami M. Lung development and function in preterm infants in the surfactant treatment era. Annu Rev Physiol 2000;62:825-46.

2. Ammari A, Suri M, Milisavljevic V, et al. Variables associated with the early failure of nasal CPAP in very low birth weight infants. J Pediatr 2005;147:341-7.

3. Horbar JD, Badger GJ, Carpenter JH, et al.; Members of the Vermont Oxford Network. Trends in mortality and morbidity for very low birth weight infants, 1991-1999. Pediatrics 2002;110(1 Pt 1):143-51. 
4. Jobe AH, Bancalari E. Bronchopulmonary dysplasia. Am J Respir Crit Care Med 2001;163:1723-9.

5. Bhat RY, LeipäläJA, Singh NR, Rafferty GF, Hannam S, Greenough A. Effect of posture on oxygenation, lung volume, and respiratory mechanics in premature infants studied before discharge. Pediatrics 2003;112(1 Pt 1):29-32.

6. Leipälä JA, Bhat RY, Rafferty GF, Hannam S, Greenough A. Effect of posture on respiratory function and drive in preterm infants prior to discharge. Pediatr Pulmonol 2003;36:295-300.

7. Wolfson MR, Greenspan JS, Deoras KS, Allen JL, Shaffer TH. Effect of position on the mechanical interaction between the rib cage and abdomen in preterm infants. J Appl Physiol (1985) 1992;72:1032-8.

8. Wagaman MJ, Shutack JG, Moomjian AS, Schwartz JG, Shaffer TH, Fox WW. Improved oxygenation and lung compliance with prone positioning of neonates. J Pediatr 1979;94:787-91.

9. Dimitriou G, Greenough A, Laubscher B. Lung volume measurements immediately after extubation by prediction of "extubation failure" in premature infants. Pediatr Pulmonol 1996;21:250-4.

10. Kavvadia V, Greenough A, Dimitriou G. Prediction of extubation failure in preterm neonates. Eur J Pediatr 2000;159:227-31.

11. Victorino JA, Borges JB, Okamoto VN, et al. Imbalances in regional lung ventilation: a validation study on electrical impedance tomography. Am J Respir Crit Care Med 2004;169:791-800.

12. Hahn G, Sipinková I, Baisch F, Hellige G. Changes in the thoracic impedance distribution under different ventilatory conditions. Physiol Meas 1995;16(3 Suppl A):A161-73.

13. Harris ND, Suggett AJ, Barber DC, Brown BH. Applied potential tomography: a new technique for monitoring pulmonary function. Clin Phys Physiol Meas 1988;9 Suppl A:79-85.

14. Harris ND, Suggett AJ, Barber DC, Brown BH. Applications of applied potential tomography (APT) in respiratory medicine. Clin Phys Physiol Meas 1987;8 Suppl A:155-65.

15. Frerichs I, Schiffmann H, Hahn G, Hellige G. Non-invasive radiation-free monitoring of regional lung ventilation in critically ill infants. Intensive Care Med 2001;27:1385-94.

16. Miedema M, de Jongh FH, Frerichs I, van Veenendaal MB, van Kaam AH. Changes in lung volume and ventilation during surfactant treatment in ventilated preterm infants. Am J Respir Crit Care Med 2011;184:100-5.

17. van der Burg PS, Miedema M, de Jongh FH, Frerichs I, van Kaam AH. Cross-sectional changes in lung volume measured by electrical impedance tomography are representative for the whole lung in ventilated preterm infants. Crit Care Med 2014;42:1524-30.

18. Gerstmann DR, Fouke JM, Winter DC, Taylor AF, deLemos RA. Proximal, tracheal, and alveolar pressures during high-frequency oscillatory ventilation in a normal rabbit model. Pediatr Res 1990;28:367-73.
19. Pillow JJ, Neil H, Wilkinson MH, Ramsden CA. Effect of I/E ratio on mean alveolar pressure during high-frequency oscillatory ventilation. J Appl Physiol (1985) 1999;87:407-14.

20. Courtney SE, Durand DJ, Asselin JM, Hudak ML, Aschner JL, Shoemaker CT; Neonatal Ventilation Study Group. High-frequency oscillatory ventilation versus conventional mechanical ventilation for very-low-birthweight infants. N Engl J Med 2002;347:643-52.

21. Harrison VC, Heese Hde V, Klein M. The significance of grunting in hyaline membrane disease. Pediatrics 1968;41:549-59.

22. Harding R. Function of the larynx in the fetus and newborn. Annu Rev Physiol 1984;46:645-59.

23. Brown BH, Barber DC, Seagar AD. Applied potential tomography: possible clinical applications. Clin Phys Physiol Meas 1985;6:109-21.

24. Gouna G, Rakza T, Kuissi E, Pennaforte T, Mur S, Storme L. Positioning effects on lung function and breathing pattern in premature newborns. J Pediatr 2013;162:1133-7, 1137.e1.

25. West JB. Respiratory Physiology: The Essentials. 6th edn. Baltimore, MD: Lippincott Williams \& Wilkins, 2000.

26. Cave P, Fletcher G. Resistance of nasotracheal tubes used in infants. Anesthesiology 1968;29:588-90.

27. LeSouef PN, England SJ, Bryan AC. Total resistance of the respiratory system in preterm infants with and without an endotracheal tube. J Pediatr 1984;104:108-11.

28. Schibler A, Yuill M, Parsley C, Pham T, Gilshenan K, Dakin C. Regional ventilation distribution in non-sedated spontaneously breathing newborns and adults is not different. Pediatr Pulmonol 2009;44:851-8.

29. Frerichs I, Schiffmann H, Oehler R, et al. Distribution of lung ventilation in spontaneously breathing neonates lying in different body positions. Intensive Care Med 2003;29:787-94.

30. Hough JL, Johnston L, Brauer SG, Woodgate PG, Pham TM, Schibler A. Effect of body position on ventilation distribution in preterm infants on continuous positive airway pressure. Pediatr Crit Care Med 2012;13: 446-51.

31. Hough JL, Johnston L, Brauer S, Woodgate P, Schibler A. Effect of body position on ventilation distribution in ventilated preterm infants. Pediatr Crit Care Med 2013;14:171-7.

32. Frerichs I, Dargaville PA, van Genderingen H, Morel DR, Rimensberger PC. Lung volume recruitment after surfactant administration modifies spatial distribution of ventilation. Am J Respir Crit Care Med 2006;174:772-9.

33. Miedema M, van der Burg PS, Beuger S, de Jongh FH, Frerichs I, van Kaam AH. Effect of nasal continuous and biphasic positive airway pressure on lung volume in preterm infants. J Pediatr 2013;162: 691-7. 